\title{
Efeitos da gestão de mobilidade urbana para os Jogos Olímpicos sobre a qualidade do ar na região central da cidade do Rio de Janeiro
}

\author{
Effects of urban mobility management for the Olympic Games on air quality in \\ the central region of the city of Rio de Janeiro
}

Ingrid do Amaral Gomes ${ }^{[a]}$, Nicole Jannarella de Carvalho ${ }^{[b]}$, Rafael Ramos da Silva $a^{[b]}$, Graciela Arbilla ${ }^{[b]}$, Cleyton Martins da Silva $a^{[a, b]}$

\author{
[a] Universidade Veiga de Almeida (UVA), Rio de Janeiro, RJ, Brasil \\ [b] Universidade Federal do Rio de Janeiro (UFRJ), Instituto de Química, Rio de Janeiro, RJ, Brasil
}

\section{Resumo}

\begin{abstract}
Neste estudo, foram analisados os possíveis efeitos das obras de mobilidade urbana, realizadas no bairro Centro da cidade do Rio de Janeiro, sobre a qualidade do ar. Foram tratados estatisticamente os dados de concentração obtidos na Estação de Monitoramento do Largo da Carioca para os poluentes $\mathrm{PM}_{10}, \mathrm{CO}_{3} \mathrm{O}_{3}$ e $\mathrm{SO}_{2}$, assim como os parâmetros meteorológicos, para os meses de julho e agosto de 2015 e 2016, respectivamente, ano que precedeu e ano em que foram realizados os Jogos Olímpicos Rio 2016. Os resultados obtidos mostram que, embora as concentrações dos poluentes monitorados não tenham, em geral, ultrapassado os padrões nacionais de qualidade do ar, os níveis de $\mathrm{PM}_{10}$ e de ozônio são preocupantes, especialmente considerando as orientações da Organização Mundial da Saúde. Os níveis maiores de ozônio em agosto de 2016 podem ser consequência do aumento da radiação solar ou ainda dos processos químicos envolvidos na sua formação e das concentrações de seus precursores, os compostos orgânicos voláteis e os óxidos de nitrogênio $\left(\mathrm{NO}_{\mathrm{x}}\right)$. Para entender e explicar tais resultados, é indispensável dispor de dados de monitoramento contínuo de $\mathrm{NO}_{\mathrm{x}}$ e hidrocarbonetos não metânicos totais. No entanto, existe uma grave deficiência da Estação do Largo da Carioca quanto à aquisição desses dados. Apesar do esforço do corpo técnico da Secretaria Municipal de Meio Ambiente da cidade do Rio de Janeiro, o investimento do município no controle da qualidade do ar é ainda insuficiente, especialmente considerando que o $\mathrm{NO}_{2}$ é um composto legislado, colocando em risco a saúde e o bem-estar da população.
\end{abstract}

Palavras-chave: Mobilidade urbana. Qualidade do ar. Monitoramento. Rio 2016. Jogos Olímpicos.

IAG é engenheira ambiental pela Universidade Veiga de Almeida, e-mail: digrin88@hotmail.com

NJC é estudante de Engenharia Química na Escola de Química, Universidade Federal do Rio de Janeiro, estudante de Iniciação Científica no Instituto de Química, Universidade Federal do Rio de Janeiro com bolsa da FAPERJ, e-mail: njcarvalho.nick@gmail.com RRS é técnico em Química, estudante de Iniciação Científica no Instituto de Química, Universidade Federal do Rio de Janeiro, e-mail: obr.rafaelramos@hotmail.com

GA é doutora em Físico-Química, pesquisadora do CNPq, professora titular do Instituto de Química, Universidade Federal do Rio de Janeiro, e-mail: gracielaiq@gmail.com

CMS é doutor em Química (especialidade em Química Ambiental), pesquisador no Instituto de Química, Universidade Federal do Rio de Janeiro, professor na Universidade Veiga de Almeida, e-mail: martins.cleyton@gmail.com 


\section{Abstract}

In this study, the possible impact on air quality, due to changes in urban mobility infrastructure, in the central District of the city of Rio de Janeiro, was analyzed. Concentration data obtained at the Monitoring Station of Largo da Carioca, for the pollutants $\mathrm{PM}_{10^{\prime}} \mathrm{CO}, \mathrm{O}_{3}$ and $\mathrm{SO}_{z^{\prime}}$ as well as meteorological parameters, were statistically analyzed for the months July and August 2015 and 2016, respectively, the year preceding and the year of the Olympic Games in Rio, 2016. The results obtained show that, despite the concentration values for the monitored pollutants being lower than national air quality requirements, the $P M_{10}$ and ozone levels were of concern, mainly when the World Health Organization guidelines are adopted. The higher ozone levels in August 2016 may be a consequence of the increase in solar radiation or, even, of the chemical processes and concentrations of its precursors, volatile organic compounds and nitrogen oxides $\left(\mathrm{NO}_{x}\right)$. In order to understand and explain these results, $\mathrm{NO}_{x}$ and non-methane hydrocarbon data from continuous monitoring is necessary. However, data acquisition proved to be inadequate. Despite technician's efforts, at the Municipal Environmental Secretary of the City of Rio de Janeiro, government investment in air quality control is still insufficient, especially considering that $\mathrm{NO}_{2}$ is a criteria pollutant, which represents a risk to population health and welfare.

Keywords: Urban mobility. Air quality. Monitoring. Rio 2016. Olympic Games.

\section{Introdução}

A qualidade do ar de determinada região está relacionada às emissões atmosféricas associadas a um complexo conjunto de fatores, tais como a topografia e as condições meteorológicas, que podem promover a dispersão, o transporte e a concentração dos poluentes atmosféricos (Brasil, 2017).

Nas últimas décadas, a intensificação das emissões atmosféricas e o surgimento de novas fontes de emissão refletiram em episódios de poluição atmosférica excessiva, ocasionando más condições de qualidade do ar e aumentando o número de morbidade e de mortalidade relacionadas a essa causa (Braga et al., 2001; Dapper et al., 2016).

Estudos epidemiológicos e clínicos têm demonstrado altos riscos de doenças respiratórias (asma, bronquite, enfisema e câncer pulmonar) e de doenças cardiovasculares em seres humanos expostos a poluentes atmosféricos, tais como ozônio, material particulado, hidrocarbonetos aromáticos, entre outros (Braga et al., 2001; Godoi et al., 2010; Valavanidis et al., 2013), ainda que em concentrações que não ultrapassem os padrões de qualidade do ar vigentes (Han \& Naeher, 2006; Brasil, 2017).

Braga et al. (2001) sugerem ainda que tais efeitos são mais graves em crianças, idosos ou indivíduos que já apresentam outras doenças respiratórias.

Estudos publicados recentemente nos Estados Unidos indicam que o número anual de mortes, vítimas de doenças causadas por poluição atmosférica ambiental, poderá duplicar para 6,6 milhões em todo o mundo até 2050 , caso novas medidas mitigadoras não sejam tomadas (Kollipara, 2015).

O aumento no número de atendimentos e de internações hospitalares e no uso de medicamentos, decorrentes dos efeitos deletérios da poluição atmosférica à saúde e à qualidade de vida humana, pode ocasionar maiores gastos ao Estado (Brasil, 2017). A Organização Mundial da Saúde revela que, atualmente, as mortes e as doenças causadas por esse tipo de poluição geram um custo de 1,6 trilhão de dólares para a Europa, valor esse quase equivalente a um décimo do produto interno bruto dessa região (ONU, 2015).

A degradação da qualidade do ar também pode proporcionar efeitos adversos sobre a fauna, a flora e os materiais, além de alterar as propriedades físicas da atmosfera (Kuklinska et al., 2015) e, assim, promover prejuízos à economia de dada região.

Dessa forma, são necessárias ações de prevenção, controle e mitigação das emissões atmosféricas, de forma a garantir uma qualidade do ar segura para os seus diversos receptores.

A gestão da qualidade do ar, com o objetivo de promover uma melhoria dele, por meio do desenvolvimento socioeconômico sustentável e ambientalmente seguro, constitui-se em um complexo desafio para as instituições de conservação ambiental de determinada região (Kuklinska et al., 2015; Brasil, 2017). 
No Brasil, em âmbito federal, a Resolução CONAMA no 03/90 define padrões primários e secundários de qualidade do ar para alguns parâmetros - partículas totais em suspensão, partículas inaláveis, dióxido de enxofre, monóxido de carbono, ozônio, dióxido de nitrogênio e fumaça. Os padrões primários e secundários são determinados a fim de garantir, respectivamente, a saúde e o bem-estar da população, bem como a preservação do meio ambiente (Brasil, 1990).

As demais esferas de governo (estadual e municipal) são responsáveis por implementar as medidas necessárias para cumprir o estabelecido pela legislação e realizar o monitoramento da qualidade do ar, podendo determinar padrões mais restritivos.

Em um ambiente tipicamente urbano, tais emissões estão diretamente relacionadas às atividades antrópicas, principalmente àquelas destinadas à geração de energia por meio da queima de combustíveis fósseis, tais como carvão e derivados de petróleo.

Dentre as emissões antropogênicas, as emissões veiculares contribuem fortemente para a poluição atmosférica, principalmente em grandes centros urbanos. o Brasil apresenta um crescimento expressivo na frota veicular de suas regiões metropolitanas, sobretudo na Região Metropolitana de São Paulo (RMSP) e na Região Metropolitana do Rio de Janeiro (RMRJ), que detêm as duas maiores frotas automotivas do país (CETESB, 2015; INEA, 2016).

Na RMSP, as emissões veiculares são responsáveis por $97 \%$ das emissões de monóxido de carbono, $82 \%$ de hidrocarbonetos, $78 \%$ de óxidos de nitrogênio, $43 \%$ de óxidos de enxofre e $40 \%$ de material particulado (CETESB, 2015), e, na RMRJ, as fontes móveis são responsáveis por $77 \%$ das emissões atmosféricas (INEA, 2016).

Kuklinska et al. (2015) enfatizam que a gestão de qualidade do ar só pode ser considerada eficaz com um monitoramento das condições atmosféricas, no que tange a obtenção de dados para a determinação da composição atmosférica e de outros parâmetros que, em consonância com a legislação vigente, estabeleça o nível de degradação da atmosfera e ainda permita a orientação e a tomada de ações.

Dessa forma, das diversas ações de gestão da qualidade do ar, possuem destaques aquelas que são destinadas ao monitoramento e à redução das emissões veiculares, tais como a melhoria das características dos combustíveis e motores, as medidas complementares de redução do número de viagens e dos congestionamentos, o aumento da oferta e da eficiência de transporte público e ainda do planejamento do uso do solo (CETESB, 2015).

Como consequência das exigências para sediarem megaeventos, diversas cidades têm promovido mudanças e um maior desenvolvimento urbano, por meio do estímulo ao crescimento econômico, da otimização da mobilidade urbana e da construção de instalações culturais (Chalkley \& Essex, 1999; Hiller, 2000), que deveriam se constituir nos legados desses acontecimentos.

Algumas dessas mudanças podem trazer reflexos diretos na qualidade do ar de uma cidade, por exemplo, instalações de parques urbanos e alterações na topografia local, mas, principalmente, aquelas que atuam nos setores de energia e mobilidade urbana - alterações no sistema de transporte público e modificações no tráfego local, como aconteceu em Atlanta-1996 (Friedman et al., 2001; Tian \& Brimblecombe, 2008), Sydney-2000 (Tian \& Brimblecombe, 2008), Busan-2002 (Lee et al., 2007), Atenas-2004 (Tian \& Brimblecombe, 2008), Pequim-2008 (Rich et al., 2012; Wang et al., 2010) e Londres-2012 (McCarthy et al., 2010; McCartney et al., 2010; Ma et al., 2008).

Nos últimos dez anos, a cidade do Rio de Janeiro recebeu quatro grandes eventos: o XV Jogos Pan-Americanos em 2007, a XXVIII Jornada Mundial da Juventude (2013), a Copa do Mundo FIFA (2014) e os Jogos da XXXI Olimpíada (2016).

Para cumprir as exigências dos organizadores de tais eventos, as diferentes esferas de governo que atuam na cidade do Rio de Janeiro se depararam com a necessidade de realizar investimentos promovendo um desenvolvimento urbano sustentável. Essas ações incluíram a mobilidade urbana e utilização do solo, a melhoria dos transportes públicos por meio da criação do Anel de Transporte de Alta Capacidade, a transformação da zona portuária em uma área residencial, de entretenimento e de turismo e a construção de novos centros residenciais e de lazer e cultura. Muitas dessas mudanças urbanas foram realizadas no centro da cidade, tanto no trânsito como na revitalização das áreas culturais e de lazer (Rio de Janeiro, 2009).

Dessa forma, este estudo visa analisar os efeitos sobre a qualidade do ar, como consequência das modificações urbanas, quanto à mobilidade urbana, que se deu no bairro Centro da cidade do Rio de Janeiro, sobretudo em 2015 e 2016, respectivamente, ano que precedeu e ano em que foram realizados os Jogos Olímpicos Rio 2016. 


\section{Local de estudo}

A cidade do Rio de Janeiro está situada na região Sudeste do Brasil e é a segunda maior cidade do país. Possui uma população de 6.498 .837 habitantes (Rio de Janeiro, 2005; IBGE, 2017) e 1.224,56 km² de área, cujo relevo contrastante se distribui por extensas áreas de baixadas, de lagunas e pela presença de três maciços montanhosos: Pedra Branca, Gericinó e Tijuca (Rio de Janeiro, 2005).

0 clima da cidade é predominantemente tropical, quente e úmido, com temperaturas médias de $23^{\circ} \mathrm{Ce} 27^{\circ} \mathrm{C}$ no inverno e no verão, respectivamente $\mathrm{A}$ temperatura média máxima é obtida no verão $\left(30,2^{\circ} \mathrm{C}\right)$, e a mínima, no inverno $\left(18,4{ }^{\circ} \mathrm{C}\right)$ (Rio de Janeiro, 2016).

No entanto, segundo Martins et al. (2014), o relevo acidentado da região, com áreas de planície circundadas por montanhas, delimita a formação de "bacias aéreas", que caracterizam-se por topografia e aspectos meteorológicos homogêneos. Nesse contexto, o município do Rio de Janeiro abrange quatro bacias aéreas distintas entre si.

Assim, compreendendo o conceito de bacias aéreas, bem como a influência meteorológica e a dispersão das fontes de emissão que atuam sobre elas, é comum se utilizar desses conceitos para a gestão e o monitoramento da qualidade do ar.

Das bacias aéreas definidas na cidade do Rio de Janeiro, a Bacia Aérea III apresenta maior destaque que as demais em razão da grande quantidade de potenciais fontes de emissões atmosféricas, tanto móveis quanto estacionárias.

Nessa região, localiza-se um significativo número de indústrias, principalmente o polo industrial de Campos Elíseos (localizado em Duque de Caxias, município vizinho ao Rio de Janeiro), e ainda é uma importante via de acesso à região central da cidade (rodovias Washington Luiz e Presidente Dutra, as Linhas Vermelha e Amarela, um trecho da Ponte Rio-Niterói e grande parte da Avenida Brasil), todas importantes vias de trânsito, contribuindo, assim, para a degradação da qualidade do ar da Bacia Aérea III (Martins et al., 2014). Sendo assim, essa região pode ser considerada prioritária para as ações de controle da gestão da qualidade do ar.

Segundo o Diagnóstico Urbano-Ambiental da Cidade do Rio de Janeiro (Rio de Janeiro, 2015), o sistema de transporte público da capital carioca e de toda a
RMRJ possui uma estrutura radial concêntrica, cujo centro é a região central do Rio de Janeiro.

Nos últimos anos, o sistema de transporte público da cidade do Rio de Janeiro sofreu grandes transformações que implicaram a alteração da sua estrutura física, como a implementação do sistema de transporte rápido (BRT) e do sistema de transporte sobre trilhos, a construção de novas vias e ciclovias e a ampliação da rede de metrô. As obras de revitalização da região portuária, a demolição do Elevado da Perimetral e a construção de novas vias e túneis modificaram também o uso e a ocupação do solo urbano da região. Tais medidas podem ter provocado efeitos sobre a qualidade do ar no município, sobretudo na região central da cidade, onde se deu grande parte das modificações.

0 bairro Centro possui cerca de 41 mil habitantes e uma área territorial de $5,72 \mathrm{~km}^{2}$ (Rio de Janeiro, 2005; IBGE, 2010). 0 seu relevo e a sua cobertura vegetal podem ser caracterizados em grande parte por terrenos de baixada e pela faixa litorânea da Baía de Guanabara nos quadrantes noroeste, nordeste e sudeste. 0 quadrante noroeste ainda engloba uma borda do Maciço da Tijuca, cujas áreas de encosta ultrapassam a cota de $100 \mathrm{~m}$ no bairro de Santa Teresa (Rio de Janeiro, 2016).

Em relação ao uso do solo, essa região é completamente ocupada por áreas antrópicas, que são subdivididas em áreas urbanas, vegetação arbóreo-arbustiva e áreas de reflorestamento. Nesse bairro, encontram-se os centros financeiro e histórico da cidade, concentrando, assim, o maior número de estabelecimentos de comércio e de serviços, além de áreas de atividade militar, portuária e aeroportuária (Aeroporto Santos Dumont), bem como os patrimônios naturais e culturais, tais como o Campo de Santana, o trecho do Aterro do Flamengo, o Passeio Público e 202 exemplares de patrimônio construído tombados (Rio de Janeiro, 2016).

0 monitoramento da qualidade do ar dessa região é realizado por uma estação, da Secretaria Municipal de Meio Ambiente (SMAC), localizada no Largo da Carioca $\left(22^{\circ} 54^{\prime} 30.02^{\prime \prime} \mathrm{S}, 43^{\circ} 10^{\prime} 41.36^{\prime \prime} 0\right)$, pertencente à rede de monitoramento da Prefeitura da cidade do Rio de Janeiro. A área de abrangência de monitoramento dessa estação atinge um raio de $2 \mathrm{~km}$, contemplando todo o bairro Centro, além de bairros adjacentes, como Glória, Saúde e, ainda parcialmente, Gamboa, Cidade Nova, Catumbi, Santa Teresa, Catete e Flamengo. Em relação às principais fontes de emissão atmosférica dessa região, destacam-se as fontes veiculares (tanto 
veículos leves quanto ônibus) que circulam no bairro em diversas vias de tráfego de grande movimentação, além de terminais rodoviários e edifícios-garagens (Rio de Janeiro, 2016).

A Figura 1 mostra a cidade do Rio de Janeiro, o bairro Centro e vizinhanças e a estação de monitoramento do Largo da Carioca. A estação Largo da Carioca é próxima à região portuária e ao Aeroporto Santos Dumont, assim como a importantes centros culturais, por exemplo, Teatro Municipal, Museu do Amanhã, Museu Histórico Nacional, Catedral Metropolitana e importantes avenidas com intenso trânsito veicular e circulação de pessoas.

\section{Coleta e tratamento dos dados}

A estação do monitoramento localizada no Largo da Carioca mede os poluentes (partículas inaláveis $\left.\left(\mathrm{PM}_{10}\right), \mathrm{CO}, \mathrm{O}_{3}\right)$ e os parâmetros meteorológicos (temperatura, umidade relativa, pressão atmosférica, radiação solar global, precipitação pluviométrica, direção e velocidade de vento). As concentrações de $\mathrm{SO}_{2}$ foram medidas apenas no ano de 2015 . Os períodos estudados foram de $1^{\circ}$ de julho a 31 de agosto dos anos de 2015 e 2016.

Os dados brutos referentes à estação de monitoramento foram fornecidos pela SMAC na forma de uma planilha de Excel, em intervalos de 10 minutos, exceto para o $\mathrm{PM}_{10}$, para o qual o intervalo foi de 1 hora. Na área próxima à estação de monitoramento, as atividades são, principalmente, comerciais e administrativas e, portanto, focalizadas no horário diurno e nos dias úteis. Por esse motivo, os dados foram tratados previamente de modo a não considerar os sábados, domingos e feriados. Posteriormente, foram selecionados os dados correspondentes ao período das $6 \mathrm{~h}$ às $18 \mathrm{~h}$.

A organização dos dados e o tratamento preliminar, usando estatística descritiva (valor médio, mediana, desvio-padrão, mínimo e máximo), foram realizados com Microsoft Excel, por meio das funções disponíveis nesse aplicativo. 0 tratamento posterior dos dados foi efetuado mediante a linguagem de programação R-Project (R Core Team, 2017), que permite a análise

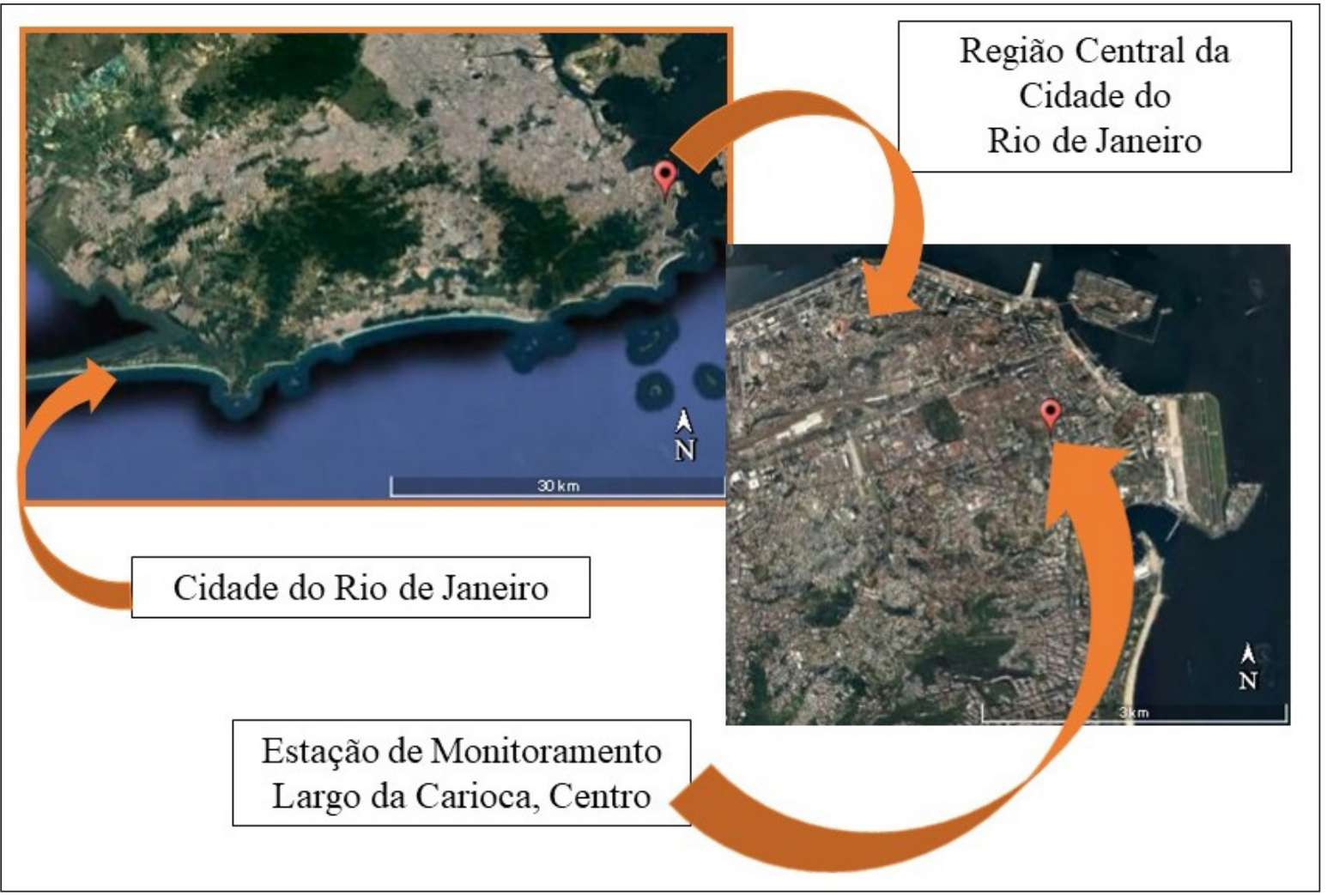

Figura 1 - Localização da Estação de Monitoramento no bairro Centro Fonte: Elaborada pelos autores a partir do Google Maps (2017). 
estatística e a construção de diferentes gráficos. O R é um software livre para estatística e construção de gráficos, que oferece recursos para diversas áreas, com uma série de comandos e funções que permitem construir diferentes gráficos, como boxplots e rosa dos ventos, além de realizar cálculos de estatística descritiva e multivariada. Neste trabalho, foi usado o ambiente R-Studio (R Core Team, 2017), por meio do qual é possível escrever códigos, com uma série de comandos que permitiram ler os dados e elaborar as diferentes figuras. Os dados foram organizados em uma planilha de Excel, no formato ${ }_{*} \mathrm{cvvs}$. Foram traçados as rosas dos ventos e os perfis de concentração dos poluentes monitorados e dos parâmetros meteorológicos para os quatro meses estudados. As figuras foram construídas na forma de boxplot, uma apresentação muito conveniente porque permite a visualização das medianas, dos valores mais prováveis (por intermédio da indicação do primeiro e terceiro quartis, Q1 e Q3, respectivamente), dos mínimos (dentro de 1,5 do menor quartil), máximos (dentro de 1,5 do maior quartil), extremos e outliers (Miller \& Miller, 2010). Neste trabalho, o tamanho da caixa é o comprimento interquartil (IQR $=$ Q3-Q1), e a mediana é indicada com uma linha preta dentro da caixa. Os whiskers se estendem entre o mínimo e o máximo, e os outliers são indicados com círculos.

Na Figura 2, é apresentado um fluxograma com o tratamento dos dados.

\section{Resultados e discussão}

\section{Rosas dos ventos}

Inicialmente, foram traçadas as rosas dos ventos para os quatro meses estudados (julho de 2015 e 2016 e agosto de 2015 e 2016), no período das $6 \mathrm{~h}$ às $18 \mathrm{~h}$, tal como apresentado na Figura 3.

A velocidade média (em m s${ }^{-1}$ ) e o percentual de calmaria foram de 1,08 e $0,7 \%, 0,93$ e $0,5 \%, 1,08$ e $9,0 \%, 1,18$ e $0,3 \%$, para julho de 2015 , julho de 2016 ,

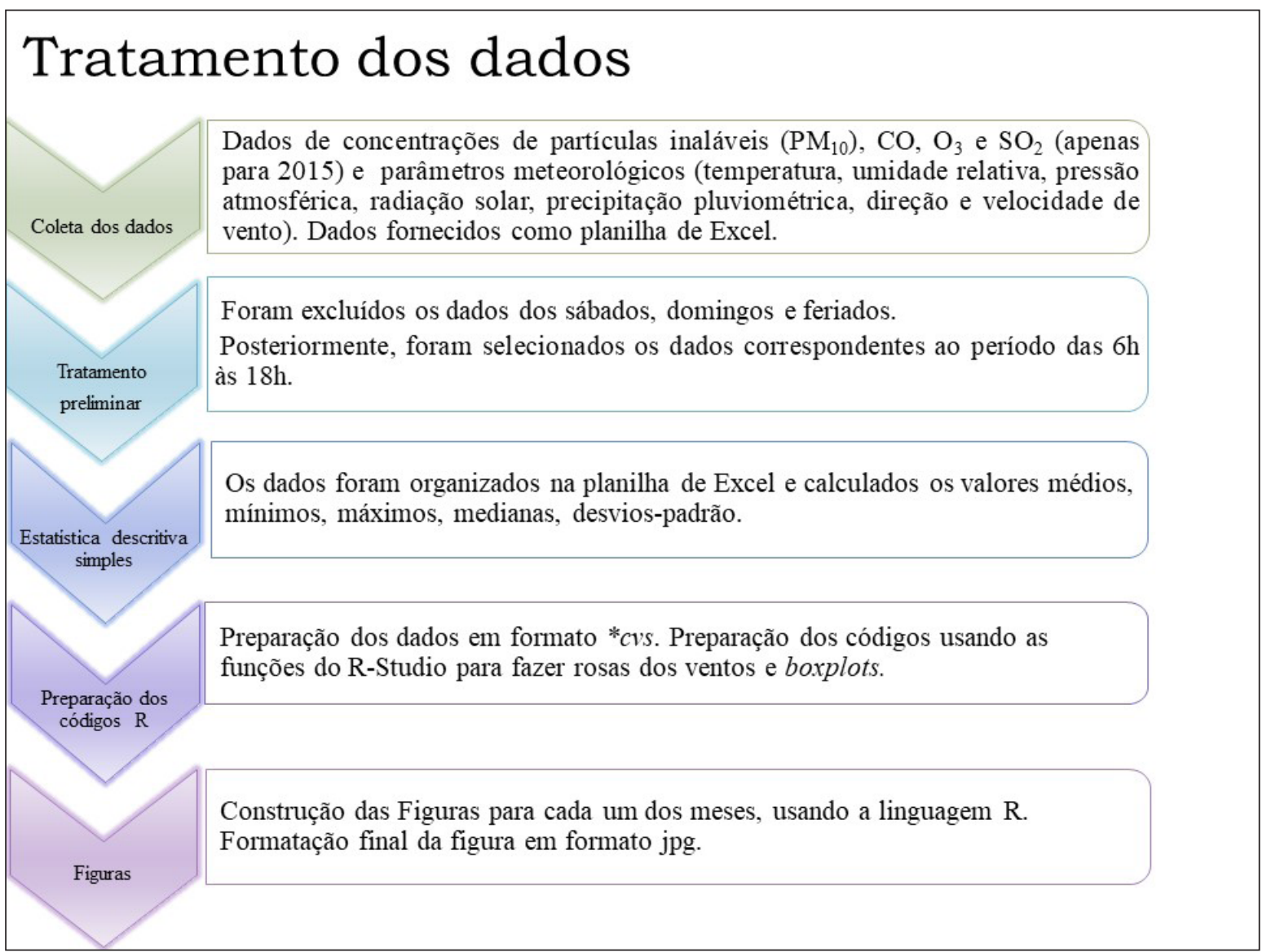

Figura 2 - Fluxograma explicativo do tratamento de dados

Fonte: Elaborada pelos autores.

urbe. Revista Brasileira de Gestão Urbana (Brazilian Journal of Urban Management), 2018, 10(Supl. 1), 129-142 


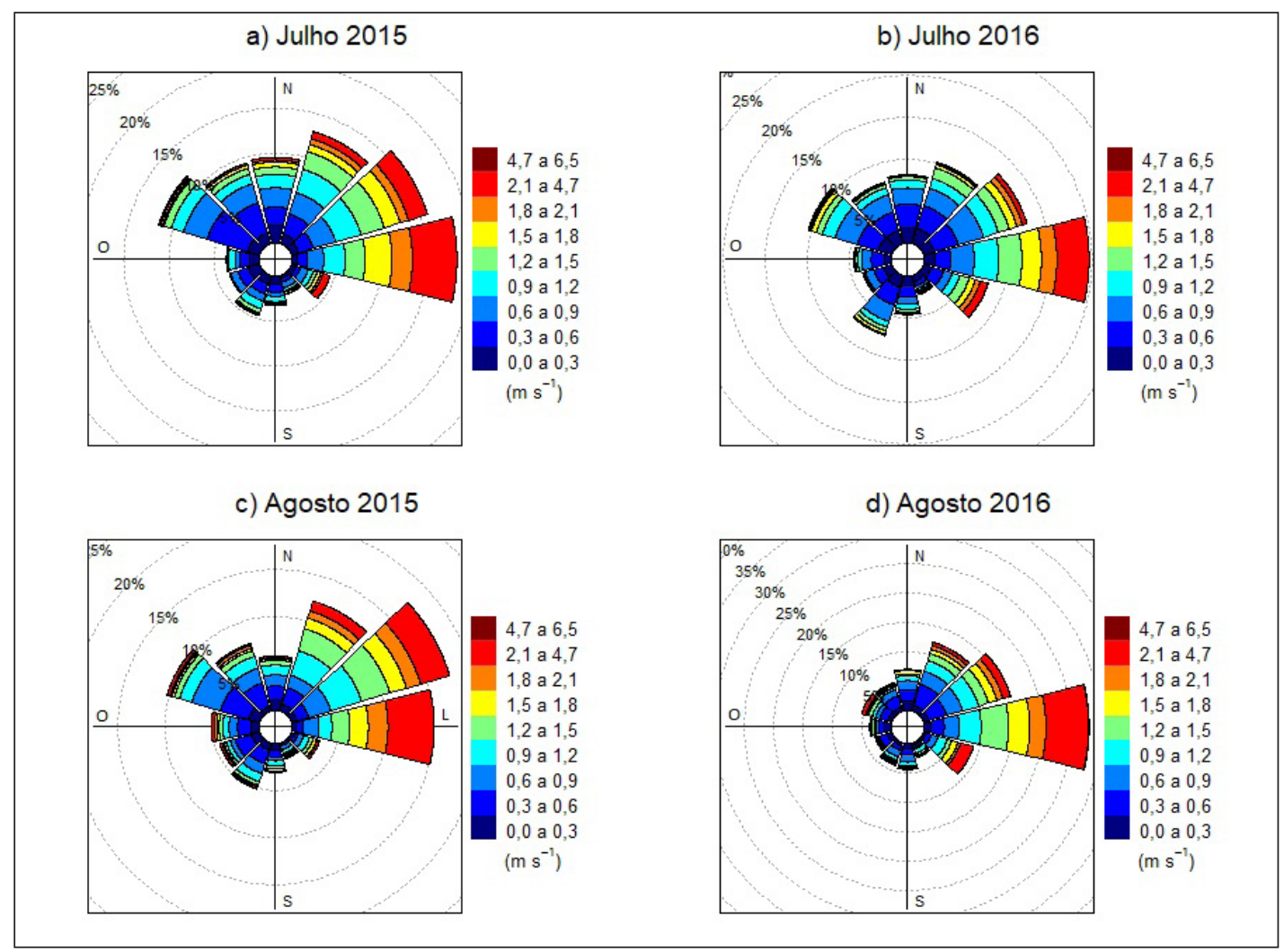

Figura 3 - Rosas dos ventos calculadas para a Estação de Monitoramento no bairro Centro para os meses de julho 2015 (a), julho 2016 (b), agosto 2015 (c) e agosto 2016 (d)

Fonte: Elaborada pelos autores.

agosto de 2015 e agosto de 2016, respectivamente. A situação de calmaria é considerada quando a velocidade do vento é inferior a $0,5 \mathrm{~m} \mathrm{~s}^{-1}$. Como já observado em trabalhos anteriores (Pimentel et al., 2014; Rio de Janeiro, 2016), os ventos são distribuídos em todas as direções, apresentando predominância dos ventos vindos da direção leste, devido à influência da brisa marítima. As características de urbanização, com edificações no entorno da estação, exercem certa influência na direção do vento. Na lateral da estação, encontra-se a Avenida Chile, que forma um corredor que se inicia na Praça do Expedicionário, próximo à Praça XV. Esse corredor, margeado por prédios altos, canaliza o vento proveniente da Baía de Guanabara, levando-o diretamente para a estação, fazendo com que haja uma segunda componente dominante na direção nordeste, alinhada com a avenida (Rio de Janeiro, 2016). Os ventos mais intensos são observados nas direções leste e nordeste, especialmente na parte da manhã.
Análise das concentrações dos polventes

Posteriormente, foram analisadas as concentrações dos poluentes monitorados nos quatro meses definidos. Para isso, foram considerados os padrões nacionais de qualidade do ar, estabelecidos por meio da Resolução CONAMA (Brasil, 1990) e apresentados na Tabela 1, e os Índices de Qualidade do Ar (IQA), determinados pela CETESB (2015) e adotados também no Estado do Rio de Janeiro (Tabela 2).

As concentrações de CO são apresentadas na Figura 4. Os padrões primário e secundário para o CO foram 9 ppm para 8 horas e 35 ppm para 1 hora (Brasil, 1990). Já o IQA utilizado no Estado de Rio de Janeiro foi Bom e Regular nas faixas 0-4,5 ppm e 4,5-9,0 ppm, respectivamente. Assim ao considerar esse poluente, tanto as medianas como os valores máximos foram muito menores que os padrões de qualidade do ar e corresponderam a um IQA Bom. Nos meses de julho de 2015 e julho de 2016, observaram-se os maiores máximos no final da tarde. Os menores valores foram 
Tabela 1 - Padrões nacionais de qualidade do ar estabelecidos por meio da Resolução CONAMA (Brasil, 1990)

\begin{tabular}{|c|c|c|c|c|c|}
\hline Parâmetros da qualidade do ar & & $\begin{array}{c}\text { Tempo de } \\
\text { amostragem }\end{array}$ & $\begin{array}{c}\text { Padrão primário } \\
\left(\mu \mathrm{g} \mathrm{m}^{-3}\right)\end{array}$ & $\begin{array}{c}\text { Padrão secundário } \\
\left(\mathrm{pg} \mathrm{m} \mathrm{m}^{-3}\right)\end{array}$ & Método de mediç̣̃o \\
\hline \multirow[t]{2}{*}{ Partículas totais em suspensão (PTS) } & 1 & CMGA & 80 & 60 & Amostrador de grandes volumes \\
\hline & 2 & CM de $24 h\left(^{\star}\right)$ & 240 & 150 & \\
\hline \multirow[t]{2}{*}{ Fumaça } & 1 & CMAA & 60 & 40 & Refletância \\
\hline & 2 & CM de $24 h\left(^{*}\right)$ & 150 & 100 & \\
\hline \multirow{2}{*}{ Partículas inaláveis (PI) ou (PM $\left.{ }_{10}\right)$} & 1 & $\mathrm{CMAA}^{(* \star)}$ & 50 & 50 & Separação inercial \\
\hline & 2 & CM de $24 h\left(^{\star}\right)(\star \star)$ & 150 & 150 & Filtração \\
\hline \multirow[t]{2}{*}{ Dióxido de enxofre $\left(\mathrm{SO}_{2}\right)$} & 1 & CMAA & 80 & 40 & Pararosanilina \\
\hline & 2 & CM de $24 h\left(^{\star}\right)$ & 365 & 100 & \\
\hline \multirow[t]{4}{*}{ Monóxido de carbono (CO) } & 1 & CM de $8 h\left({ }^{\star}\right)\left({ }^{\star \star}\right)$ & 10 & 10 & Infravermelho não dispersivo \\
\hline & & & (9 ppm) & (9ppm) & \\
\hline & 2 & CM de $\operatorname{lh}\left({ }^{\star}\right)\left({ }^{\star \star}\right)$ & 40 & 40 & \\
\hline & & & (35 ppm) & (35ppm) & \\
\hline Ozônio $\left(\mathrm{O}_{3}\right)$ & 1 & CM de $\operatorname{lh}\left({ }^{*}\right)(* \star)$ & 160 & 160 & Quimiluminescência \\
\hline \multirow[t]{2}{*}{ Dióxido de nitrogênio $\left(\mathrm{NO}_{2}\right)$} & 1 & CMAA & 100 & 100 & Quimiluminescência \\
\hline & 2 & CM de lh & 320 & 190 & \\
\hline
\end{tabular}

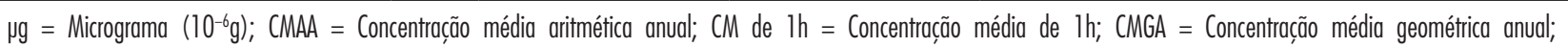
CM de $8 \mathrm{~h}$ = Concentração média de 8h; $C M$ de 24h = Concentraccão média de 24h; ${ }^{*}$ ) Não deve ser excedida mais de uma vez por ano; ${ }^{* \star}$ ) Os valores de padrão primário e padrão secundário são iguais. Fonte: Elaborada a partir da Resoluc̦ão CONAMA no 03, de 28 de junho de 1990, Art $3^{\circ}$ (Brasil, 1990).

Tabela 2 - Intervalo de classificaç̃õo das concentraç̃oes dos poluentes para cálculo do Índice de Qualidade do Ar (IQA)

\begin{tabular}{|c|c|c|c|c|c|c|}
\hline Qualidade do ar & IQA & $\mathrm{CO}$ (ppm) & $\frac{\mathrm{SO}_{2}}{\left(\mathrm{\mu g} \mathrm{m}^{-3}\right)}$ & $\frac{\mathrm{NO}_{2}}{\left(\mathrm{pg} \mathrm{m}^{-3}\right)}$ & $\frac{\mathbf{O}_{3}}{\left(\mathrm{pg} \mathrm{m}^{-3}\right)}$ & $\frac{\mathrm{PM}_{10}}{\left(\mathrm{pg} \mathrm{m}^{-3}\right)}$ \\
\hline Boa & $0-50$ & $0 \leq e<4,5$ & $0 \leq e<80$ & $0 \leq e<100$ & $0 \leq e<80$ & $0 \leq e<50$ \\
\hline Regular & $51-100$ & $4,5 \leq e<9$ & $80 \leq e<365$ & $100 \leq e<320$ & $80 \leq e<160$ & $50 \leq e<150$ \\
\hline Inadequada & $101-199$ & $9 \leq e<15$ & $365 \leq e<800$ & $320 \leq e<1130$ & $160 \leq e<200$ & $150 \leq e<250$ \\
\hline Má & $200-299$ & $15 \leq e<30$ & $800 \leq e<1600$ & $1130 \leq e<2260$ & $200 \leq e<800$ & $250 \leq e<420$ \\
\hline Péssima & $>299$ & $>30$ & $>1600$ & $>2260$ & $>800$ & $>420$ \\
\hline
\end{tabular}

Fonte: Elaborada a partir de MonitorA-Rio $2011-2012$ (Rio de Janeiro, 2016).

observados em agosto de 2015, enquanto os maiores valores, especialmente máximos, foram percebidos em agosto de 2016.

As concentrações horárias de material particulado

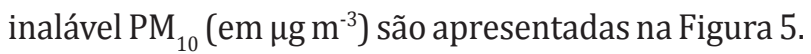
0 valor de $50 \mu \mathrm{g} \mathrm{m}^{-3}$ indicado na figura correspondeu ao IQA Regular e coincidiu com a média de 24 horas estabelecida pela União Europeia e recomendada pela Organização Mundial da Saúde.
Tanto as medianas como os valores máximos de concentração de $\mathrm{PM}_{10}$ foram menores que o padrão de qualidade do ar para 24 horas. Porém, o valor de $50 \mathrm{\mu g} \mathrm{m}^{-3}$ (que correspondia ao IQA Regular) foi superado em todos os meses. Pode ser observado que as concentrações nos meses de julho e agosto de 2015 foram, na maior parte dos horários, menores que nos mesmos meses de 2016. No ano de 2016, em geral, as menores concentrações (medianas) foram 


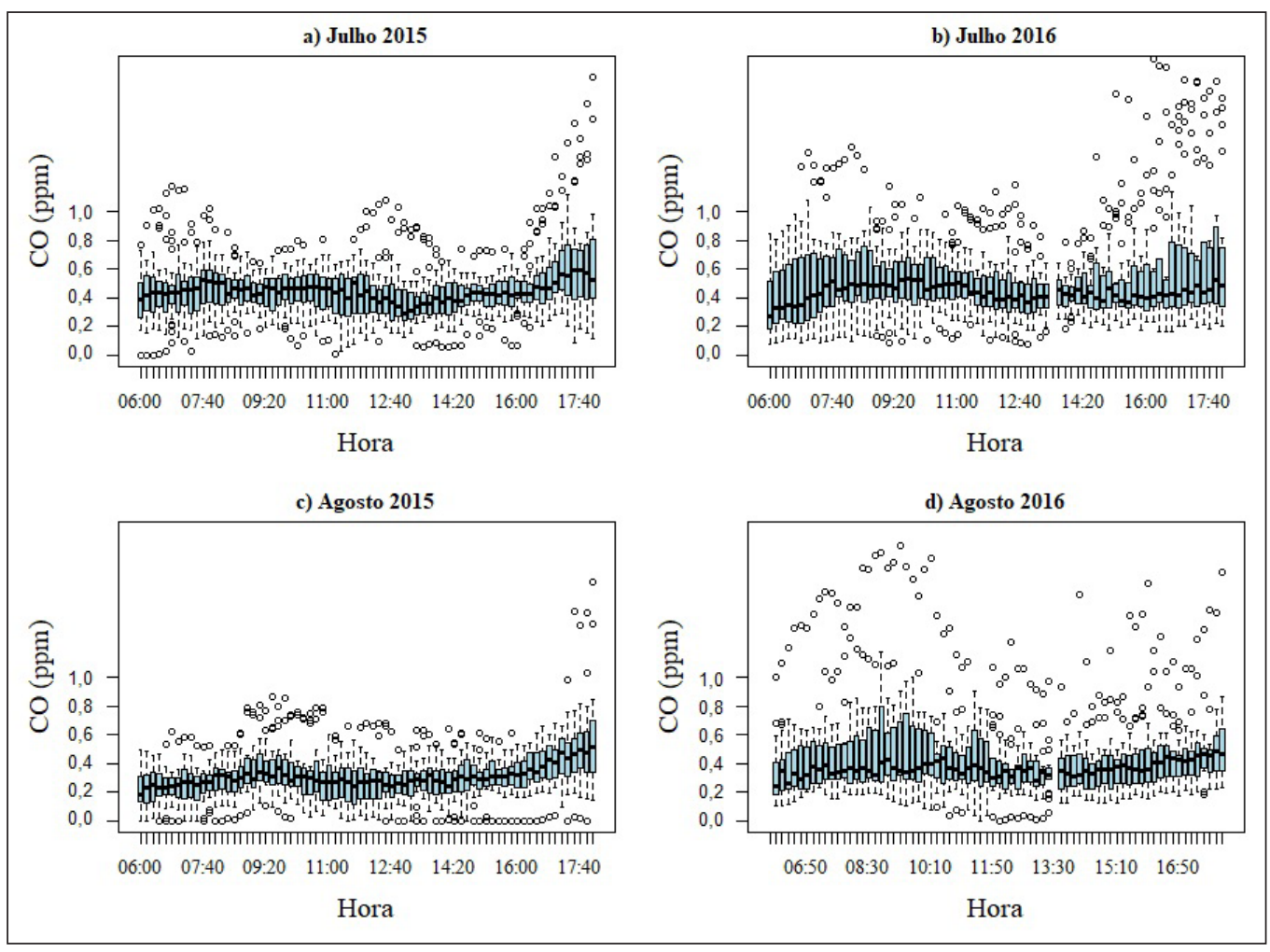

Figura 4 - Concentraç̃ões de CO (em ppm) determinadas na Estação de Monitoramento no bairro Centro para os meses de julho 2015 (a), julho 2016 (b), agosto 2015 (c) e agosto 2016 (d)

Fonte: Elaborada pelos autores.

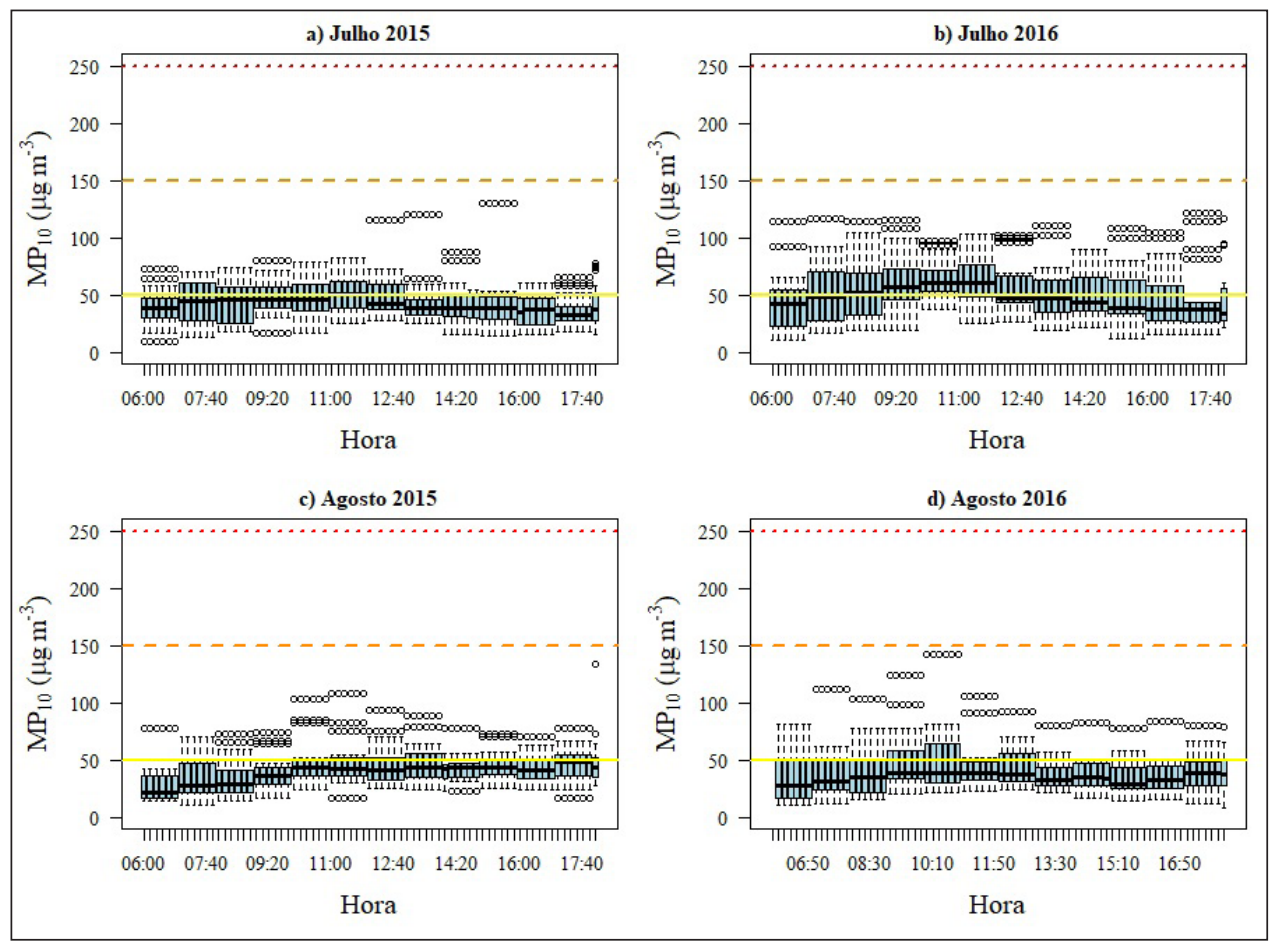

Figura 5 - Concentrações de PM 10 (em $\mu g \mathrm{~m}^{3}$ ) determinadas na Estação de Monitoramento no bairro Centro para os meses de julho 2015 (a), julho 2016 (b), agosto 2015 (c) e agosto 2016 (d). Os valores de 50, 150 e 250 $\mathrm{ug} \mathrm{m}^{3}$ correspondem aos IQA Regular, Inadequado e Mau, respectivamente Fonte: Elaborada pelos autores. 
encontradas no mês agosto, no qual foram realizados os Jogos Olímpicos.

É importante notar que, como já mencionado, a média de 24 horas estabelecida pela União Europeia e recomendada pela Organização Mundial da Saúde é de $50 \mu \mathrm{g} \mathrm{m}^{-3}$; dessa forma, a qualidade do ar, em comparação com esses parâmetros internacionais, não foi satisfatória (Kuklinska et al., 2015).

As concentrações de $\mathrm{SO}_{2}$ apenas foram monitoradas em 2015 porque o equipamento da Estação de Monitoramento do Largo da Carioca apresentou defeito e não foi substituído. Nos dois meses estudados, as concentrações foram sempre menores que os padrões, correspondendo a um IQA Bom. Em geral, as concentrações de $\mathrm{SO}_{2}$ na cidade do Rio de Janeiro foram menores que os padrões nacionais de qualidade do ar em todas as estações de monitoramento (Rio de Janeiro, 2016; INEA, 2016) e não representaram uma preocupação ambiental e para a saúde dos seres vivos. Contudo, por ser um poluente legislado, é importante a substituição do equipamento, uma vez que, neste estudo, os dados referentes a esse poluente não demonstraram uma criticidade para a qualidade do ar; além disso, pela indisponibilidade dos dados para o ano de 2016, as figuras não são apresentadas neste trabalho.

Já as concentrações de ozônio (em unidades de $\mu \mathrm{g} \mathrm{m}^{-3}$ ) são demonstradas na Figura 6. Na Figura 6a, é indicado o valor de $80 \mu \mathrm{g} \mathrm{m}^{-3}$, que correspondeu ao IQA Regular, e na Figura 6d, o valor de $160 \mathrm{\mu g} \mathrm{m}^{-3}$, que correspondeu ao IQA Inadequado.

As concentrações medianas de ozônio para 1 hora permaneceram na faixa de IQA Bom, porém as médias de 1 hora para alguns horários ultrapassaram o valor de $80 \mu \mathrm{g} \mathrm{m}^{-3}$, especialmente no ano de 2016. Por ser um poluente secundário, formado por meio da oxidação fotoquímica dos Compostos Orgânicos Voláteis (COVs) na presença de óxidos de nitrogênio, $\mathrm{NO}+\mathrm{NO}_{2},\left(\mathrm{NO}_{\mathrm{x}}\right)$, em geral, o máximo para as concentrações se encontra aproximadamente a 1 PM e 3 PM. Os menores valores

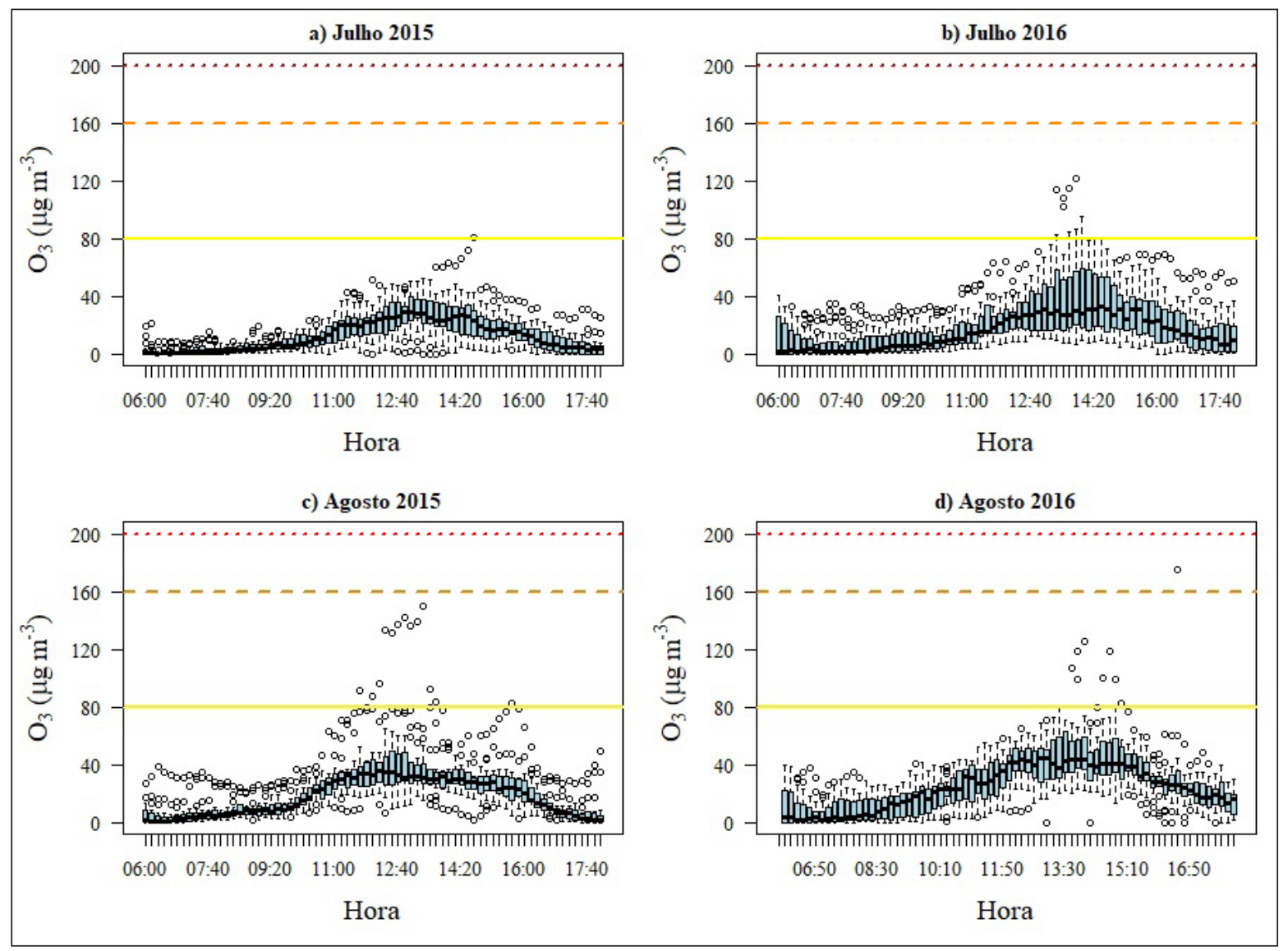

Figura 6 - Concentraç̃oes de ozônio (em $\mu g$ m $^{3}$ ) determinadas na Estação de Monitoramento no bairro Centro para os meses de julho 2015 (a), julho 2016 (b), agosto 2015 (c) e agosto 2016 (d). As linhas correspondentes aos valores 80, 160 e $200 \mathrm{jg} \mathrm{m}^{-3}$ se referem aos IQA Regular, Inadequado e Mau, respectivamente Fonte: Elaborada pelos autores. 


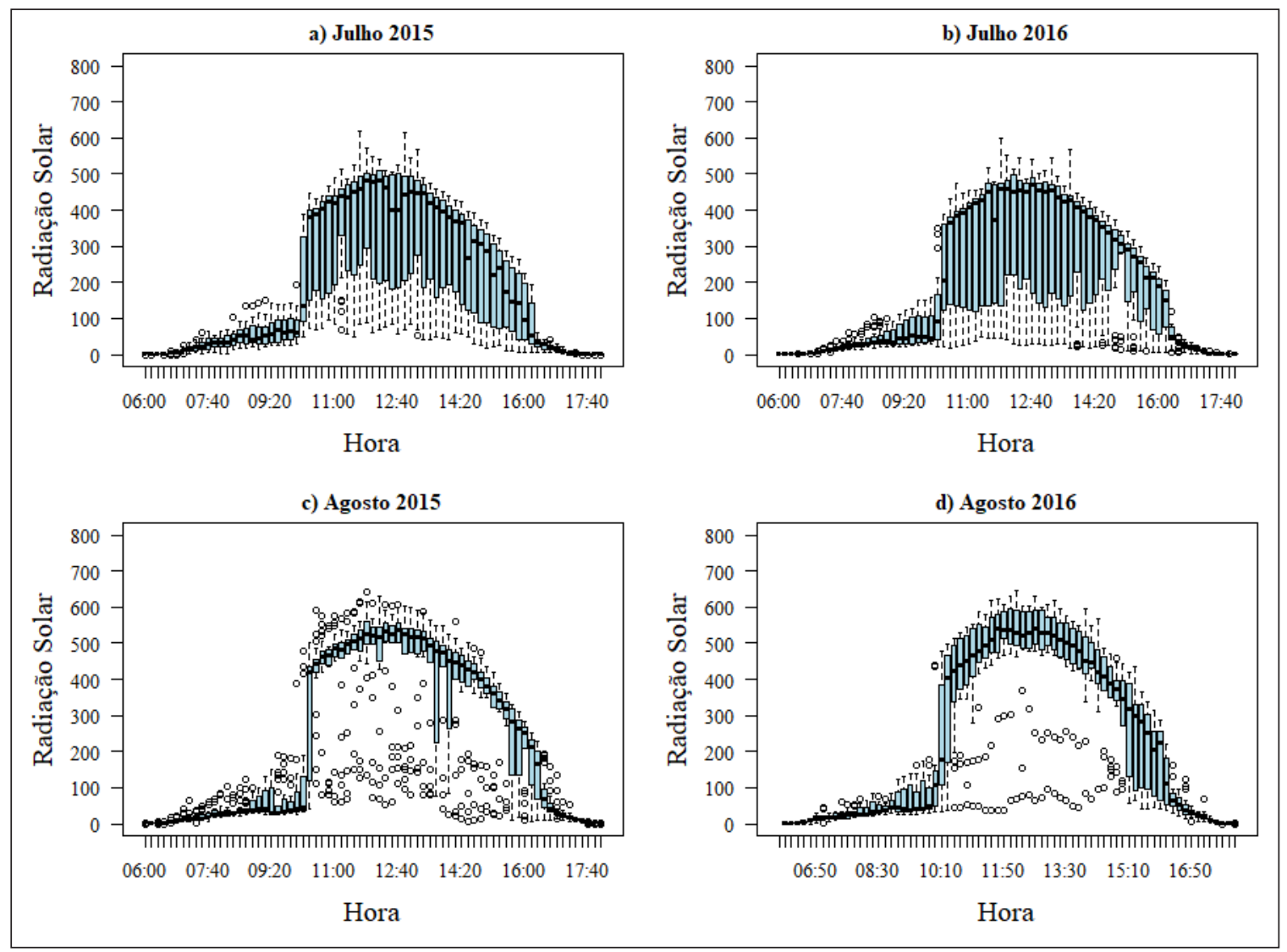

Figura 7 - Radiação solar (em W m³) determinada na Estação de Monitoramento no bairro Centro para os meses de julho 2015 (a), julho 2016 (b), agosto 2015 (c) e agosto 2016 (d)

Fonte: Elaborada pelos autores.

para as medianas foram para julho de 2015 e 2016, não tendo uma diferença significativa entre julho 2015-2016. Os valores para agosto foram os maiores que os de julho e ainda maiores em 2016.

Os valores máximos das medianas para as concentrações de $\mathrm{O}_{3}$ nos meses de julho e agosto de 2015 foram de 28,84 e $35,42 \mu \mathrm{g} \mathrm{m}^{-3}$, respectivamente. Sendo assim, o valor para o mês de agosto foi $22,8 \%$ maior que o de julho. Já para o ano de 2016, tais valores máximos para as medianas das concentrações de $\mathrm{O}_{3}$ para os meses de julho e agosto foram 33,13 e 44,83 $\mathrm{\mu g} \mathrm{m}^{-3}$, respectivamente. Dessa forma, o valor para o mês de agosto foi 35,3\% maior que o de julho. Portanto, o acréscimo de concentração do mês de julho para agosto foi maior em 2016.

Considerando que o ozônio é formado mediante um processo de fotoxidação e que, por esse motivo, suas concentrações dependem da temperatura e da radiação solar (Arbilla et al., 2002), foram verificados os valores de temperaturas e radiação solar dos meses estudados. A estação não obteve registros de temperatura para os meses de julho e agosto de 2016, motivo pelo qual não foi possível comparar os anos de 2015 e 2016. Quanto à radiação solar, é possível observar que os valores das medianas para julho foram menores do que para agosto, havendo uma diferença muito pequena de um ano para outro, conforme demonstrado na Figura 7.

Entre julho de 2015 e agosto de 2015, a diferença entre os valores de mediana da radiação foi de 10,5\%; já entre os meses de julho e agosto de 2016, de 14,2\%. Além disso, nos meses de julho, houve um maior número de dias nublados, nos quais os valores de radiação estavam fora do intervalo de $50 \%$ da mediana. Assim, o aumento de concentração de ozônio em agosto pode ser devido à maior incidência de radiação solar que promove a formação desse poluente.

Como também apresentado na Figura 7, ao comparar os meses de agosto de 2015 e agosto de 2016, houve uma maior incidência de dias com radiação solar mais 
intensa em agosto de 2016 e com uma mediana da radiação solar (para o horário de máxima insolação) ligeiramente maior que a do mês de agosto de 2015 .

É possível observar ainda que o valor máximo de concentração de ozônio para agosto de 2016 foi $26,6 \%$ maior que agosto de 2015 , conforme já demonstrado na Figura 6.

No entanto, é possível que, além do efeito da radiação solar, outros fatores estejam influenciando as concentrações de ozônio. Outros aspectos deveriam ser considerados, especialmente as concentrações dos precursores da formação de ozônio (COVs e $\mathrm{NO}_{\mathrm{x}}$ ), que não estavam sendo monitorados na estação. Em geral, é observado que, em áreas urbanas, a formação de ozônio é limitada pelas concentrações e pela especiação dos compostos orgânicos voláteis, assim como maiores concentrações de $\mathrm{NO}_{x}$ podem levar ao consumo de ozônio por meio da reação com NO (Silva et al., 2016a, 2016b).

Dessa forma, eventualmente as obras de mobilidade urbana e a realização dos Jogos Olímpicos em agosto de 2016, com a consequente mudança no trânsito de pessoas e veículos, poderiam levar a uma alteração nos processos químicos de formação e de consumo de ozônio. Essa hipótese só poderia ser verificada com o monitoramento contínuo de todos os parâmetros meteorológicos, bem como das concentrações de ozônio, $\mathrm{NO}_{\mathrm{x}}$ e COVs.

\section{Conclusões}

Os resultados obtidos neste trabalho mostram que, ainda que as concentrações dos poluentes monitorados não tenham, em geral, ultrapassado os padrões nacionais de qualidade do ar, os níveis de $\mathrm{PM}_{10}$ e de ozônio são preocupantes, especialmente considerando as orientações da Organização Mundial da Saúde.

Os níveis maiores de ozônio em agosto de 2016 podem ser consequência dos processos químicos envolvidos na sua formação e das relações COVs/ $\mathrm{NO}_{\mathrm{x}}$. Para entender e explicar esses resultados, é indispensável dispor de dados de monitoramento contínuo de $\mathrm{NO}_{\mathrm{x}} \mathrm{e}$ hidrocarbonetos não metânicos (HCNM) totais, uma grave deficiência da Estação do Largo da Carioca. Apesar do esforço do corpo técnico da Secretaria Municipal de Meio Ambiente da cidade do Rio de Janeiro (SMAC), o investimento do município no controle da qualidade do ar é ainda insuficiente, especialmente considerando que o $\mathrm{NO}_{2}$ é um composto legislado.

As obras de planejamento e de modificações urbanas realizadas nos últimos anos para sediar, especialmente, os Jogos Olímpicos tiveram um efeito positivo do ponto de vista estético, cultural e turístico, mas aparentemente não levaram a uma melhoria da qualidade do ar, apesar de ter sido um dos objetivos da agenda sustentável da Candidatura Rio 2016.

\section{Referências}

Arbilla, G., Martins, E. M., Moreira, A., \& Moreira, L. F. (2002). Ozone air quality modeling. A case study: A heavily vehicle impacted urban avenue in Rio de Janeiro, Brazil. Journal of the Brazilian Chemical Society, 13(3), 308-317. http://dx.doi.org/10.1590/S0103-50532002000300004.

Braga, A., Pereira, L. A. A., Böhm, G. M., \& Saldiva, P. (2001). Poluição atmosférica e saúde humana. Revista USP, 51(51), 58-71. http://dx.doi.org/10.11606/issn.2316-9036. v0i51p58-71.

Brasil. Conselho Nacional do Meio Ambiente - CONAMA. (1990, 22 de agosto). Resolução CONAMA n. 3, de 28 de junho de 1990. Dispõe sobre padrões de qualidade do ar, previstos no PRONAR. Brasília: Diário Oficial da União. Recuperado em 10 de janeiro de 2017, de http://www.mma. gov.br/port/conama/res/res90/res0390.html

Brasil. Ministério do Meio Ambiente - MMA. (2017). Qualidade do ar. Brasília: MMA. Recuperado em 15 de janeiro de 2017, de http://www.mma.gov.br/cidades-sustentaveis/ qualidade-do-ar

Chalkley, B., \& Essex, S. (1999). Urban development through hosting international events: a history of the Olympic Games. Planning Perspectives, 14(4), 369-394. http:// dx.doi.org/10.1080/026654399364184.

Companhia Ambiental do Estado de São Paulo - CETESB. (2015). Qualidade do ar no Estado de São Paulo. São Paulo: CETESB. Recuperado em 19 de maio de 2016, de http:// ar.cetesb.sp.gov.br/wp-content/uploads/sites/37/2013/12/ RQAR-2015.pdf

Dapper, S. N., Spohr, C., \& Zanini, R. R. (2016). Poluição do ar como fator de risco para a saúde: uma revisão sistemática no estado de São Paulo. Estudos Avançados, 30(86), 83-97. http://dx.doi.org/10.1590/S0103-40142016.00100006. 
Friedman, M. S., Powell, K. E., Hutwagner, L., Graham, L. M., \& Teague, W. G. (2001). Impact of changes in transportation and commuting behaviors during the 1996 Summer Olympic Games in Atlanta on air quality and childhood asthma. Journal of the American Medical Association, 285(7), 897-905. http://dx.doi.org/10.1001/ jama.285.7.897. PMid:11180733.

Godoi, A. F. L., Godoi, R. H. M., Azevedo, R., \& Maranho, L. T. (2010). Poluição e a densidade de vegetação: BTEX em algumas áreas públicas de Curitiba-PR, Brasil. Química Nova, 33(4), 827-833. http://dx.doi.org/10.1590/ S0100-40422010000400012.

Google Maps. (2017). Recuperado em 16 de janeiro de 2017, de https://www.google.com.br/maps/place/ Centro,+Rio+de+Janeiro+-+RJ/@-22.9005452,-43.3181944,11z/ data $=$ !4m5!3m4!1s0x997f5e151c7217:0x7ffdf9c2fc30b9 $7 \mathrm{~d} ! 8 \mathrm{~m} 2 ! 3 \mathrm{~d}-22.9070828 ! 4 \mathrm{~d}-43.1819148$

Han, X., \& Naeher, L. P. (2006). A review of traffic-related air pollution exposure assessment studies in the developing world. Environment International, 32(1), 106-120. http:// dx.doi.org/10.1016/j.envint.2005.05.020. PMid:16005066.

Hiller, H. H. (2000). Mega-events, urban boosterism and growth strategies: an analysis of the objectives and legitimations of the Cape Town 2004 Olympic Bid. International Journal of Urban and Regional Research, 24(2), 449-458. http:// dx.doi.org/10.1111/1468-2427.00256.

Instituto Brasileiro de Geografia e Estatística - IBGE. (2010). Censo Demográfico 2010. Rio de Janeiro: IBGE. Recuperado em 15 de janeiro de 2017, de http://portalgeo. rio.rj.gov.br/bdario

Instituto Brasileiro de Geografia e Estatística - IBGE. (2017). Cidades. Rio de Janeiro: IBGE. Recuperado em 15 de janeiro de 2017, de http://cidades.ibge.gov.br/xtras/perfil. php? codmun $=330455$

Instituto Estadual do Ambiente - INEA. Departamento de Planejamento Ambiental. Divisão de Qualidade do Ar. (2016). Relatório anual de qualidade do ar 2015. Rio de Janeiro: INEA. Recuperado em 9 de janeiro de 2017, de http://200.20.53.3:8081/cs/groups/public/@inter_ dimfis_gear/documents/document/zwew/mtmx/ edisp/ inea0131852.pdf

Kollipara, P. (2015). Unless nations act, air pollution deaths will double by 2050. Washington: Science. Recuperado em 12 de janeiro de 2017, de http://www.sciencemag.org/ news/2015/09/unless-nations-act-air-pollution-deathswill-double-2050-study-concludes

Kuklinska, K., Wolska, L., \& Namiesnik, J. (2015). Air quality policy in the US and the EU. A review. Atmospheric Pollution Research, 6(1), 129-137. http://dx.doi.org/10.5094/ APR.2015.015.

Lee, J. T., Son, J. Y., \& Cho, Y. S. (2007). Benefits of mitigated ambient air quality due to transportation control on childhood asthma hospitalization during the 2002 Summer Asian Games in Busan, Korea. Journal of the Air \& Waste Management Association, 57(8), 968-973. http://dx.doi. org/10.3155/1047-3289.57.8.968. PMid:17824287.

Ma, Y., Richards, M., Ghanem, M., Guo, Y., \& Hassard, J. (2008). Air pollution monitoring and mining based on sensor grid in London. Sensors, 8(6), 3601-3623. http:// dx.doi.org/10.3390/s80603601. PMid:27879895.

Martins, E. M., Fortes, J. D. N., Ribeiro, G. P., \& Pereira, M. F. M. (2014). Utilização de Sistema de Informação Geográfica como ferramenta para gestão do monitoramento da qualidade do ar na Região Metropolitana do Rio de Janeiro. Engenharia Sanitaria e Ambiental, 19(spe), 43-50. http:// dx.doi.org/10.1590/S1413-41522014019010001237.

McCarthy, M., Ravelli, R. J., \& Sinclair-Williams, M. (2010). Health impact assessment of the 2012 London Olympic transport plans. European Journal of Public Health, 20(6), 619-624. http://dx.doi.org/10.1093/eurpub/ckp154. PMid:20360031.

McCartney, G., Thomas, S., Thomson, H., Scott, J., Hamilton, V., Hanlon, P., Morrison, D. S., \& Bond, L. (2010). The health and socioeconomic impacts of major multi-sport events: Systematic review (1978-2008). BMJ, 340(4), c2369. http://dx.doi.org/10.1136/bmj.c2369. PMid:20488915.

Miller, J. N., \& Miller, J. C. (2010). Statistics and chemometrics for analytical chemistry (6th ed.). London: Prentice Hall.

Organização das Nações Unidas no Brasil - ONU. (2015). ONU: estudo mostra que poluição do ar custa 1,6 trilhão de dólares por ano aos países da Europa. ONU Brasil. Recuperado em 12 de janeiro de 2017, de https://nacoesunidas.org/ onu-estudo-mostra-que-poluicao-do-ar-custa-16-trilhaode-dolares-por-ano-aos-paises-da-europa/

Pimentel, L. C. G., Marton, E., Silva, M. S., \& Jourdan, P. (2014). Caracterização do regime de vento em superfície na Região Metropolitana do Rio de Janeiro. Engenharia Sanitaria e Ambiental, 19(2), 121-132. http://dx.doi. org/10.1590/S1413-41522014000200003. 
R Core Team. (2017). The R Project for Statistical Computing. Vienna: R Foundation for Statistical Computing. Recuperado em 15 de janeiro de 2017, de http://www.r-project.org/

Rich, D. Q., Kipen, H. M., Huang, W., Wang, G., Wang, Y., Zhu, P., Ohman-Strickland, P., Hu, M., Philipp, C., Diehl, S. R., Lu, S. E., Tong, J., Gong, J., Thomas, D., Zhu, T., \& Zhang, J. J. (2012). Association between changes in air pollution levels during the Beijing Olympics and biomarkers of inflammation and thrombosis in healthy young adults. Journal of the American Medical Association, 307(19), 2068-2078. http://dx.doi.org/10.1001/jama.2012.3488. PMid:22665106.

Rio de Janeiro. Instituto Municipal de Urbanismo Pereira Passos - IPP. Secretaria Municipal de Urbanismo. Secretaria Municipal de Meio Ambiente. Departamento de Tecnologia e Informação. (2005). Indicadores ambientais da cidade do Rio de Janeiro: Brasil 2005. Rio de Janeiro: IPP. Recuperado em 16 de outubro de 2016, de http://portalgeo. rio.rj.gov.br/protocolo/Indicadores_capitulos/\%5B00IA_indicadores_2006_completo\%5D.pdf

Rio de Janeiro. (2009). Dossiê de candidatura. Rio de Janeiro: APO. Recuperado em 15 de janeiro de 2017, de http://www.apo. gov.br/index.php/matriz/a-matriz-e-o-dossie-de-candidatura/

Rio de Janeiro. Secretaria Municipal de Urbanismo SMU. (2015). Diagnóstico urbano-ambiental da cidade do Rio de Janeiro (2014-2015). Rio de Janeiro: SMU. Recuperado em 12 de janeiro de 2017, de http://www. rio.rj.gov.br/dlstatic/10112/6028774/4158532/ DiagnosticoUrbanoCTPD2015.pdf

Rio de Janeiro. Secretaria Municipal de Meio Ambiente SMAC. (2016). Qualidade do ar na cidade do Rio de Janeiro: relatório da Rede MonitorAr-Rio 2011-2012. Rio de Janeiro: SMAC. Recuperado em 1 de dezembro de 2016, de http:// www.rio.rj.gov.br/dlstatic/10112/3252594/4114836/

RelatorioMonitorar20112012.pdf

Silva, C. M., Silva, L. L., Corrêa, S. M., \& Arbilla, G. (2016a). Kinetic and mechanistic reactivity. Isoprene impact on ozone levels in an urban area near Tijuca Forest, Rio de Janeiro. Bulletin of Environmental Contamination and Toxicology, 97(6), 781-785. http://dx.doi.org/10.1007/ s00128-016-1949-6. PMid:27738711.

Silva, C. M., Souza, E. C. C. A., Silva, L. L., Oliveira, R. L., Corrêa, S. M., \& Arbilla, G. (2016b). Volatile organic compounds in the atmosphere of the Botanical Garden of the City of Rio de Janeiro. Bulletin of Environmental Contamination and Toxicology, 97(5), 653-658. http://dx.doi.org/10.1007/ s00128-016-1887-3. PMid:27416862.

Tian, Q. W., \& Brimblecombe, P. (2008). Managing air in Olympic cities. American Journal of Environmental Sciences, 4(5), 439-444. http://dx.doi.org/10.3844/ ajessp.2008.439.444.

Valavanidis, A., Vlachogianni, T., Fiotakis, K., \& Loridas, S. (2013). Pulmonary oxidative stress, inflammation and cancer: respirable particulate matter, fibrous dusts and ozone as major causes of lung carcinogenesis through reactive oxygen species mechanisms. International Journal of Environmental Research and Public Health, 10(9), 3886-3907. http://dx.doi.org/10.3390/ijerph10093886. PMid:23985773.

Wang, S., Zhao, M., Xing, J., Wu, Y., Zhou, Y., Lei, Y., He, K., Fu, L., \& Hao, J. (2010). Quantifying the air pollutants emission reduction during the 2008 Olympic Games in Beijing. Environmental Science \& Technology, 44(7), 2490-2496. http://dx.doi.org/10.1021/es9028167. PMid:20222727.

Recebido: Jun. 30, 2017

Aprovado: Fev. 17, 2018 